\title{
Circulating Tumor Cells and Cardiac Metastasis from Esophageal Cancer: A Case Report
}

\author{
Francesca Consoli $^{a}$ Giuseppina Arcangeli ${ }^{a}$ \\ Vittorio Ferrari ${ }^{a}$ Salvatore Grisanti ${ }^{a} \quad$ Camillo Almici ${ }^{b}$ \\ Tania Bordonalic Edda Simoncinia
}

aDepartment of Medical Oncology, Beretta Foundation, bepartment of Transfusion Medicine and Immunohaematology, and 'Department of Cardiology, Azienda Spedali Civili, Brescia, Italy

\section{Key Words}

Circulating tumor cell · Esophageal cancer · Heart metastasis

\begin{abstract}
We report the case of a 67-year-old man affected by metastatic esophageal cancer. The patient developed a symptomatic heart metastasis presenting as mimicking ST-segment elevation myocardial infarction. Cardiac magnetic resonance imaging (MRI) documented the presence of a mass in the apex and septum of the left ventriculum. The dissemination of cancer was confirmed by the detection of circulating tumor cells (CTCS) in the peripheral blood, measured by the CellSearch System (Veridex, LLC, Raritan, N.J., USA). The blood sample drawn at cardiac disease progression revealed the presence of 2 CTCs per $7.5 \mathrm{ml}$ of blood. This report highlights the potential role of CTCs as markers of metastatic spread.
\end{abstract}

\section{Introduction}

Metastatic squamous esophageal cancer is associated with a poor prognosis and median overall survival is 12 months [1]. The main risk factors are tobacco and alcohol abuse. Chemotherapy is not known to provide any advantage in terms of survival but may improve quality of life (QOL) in selected patients, owing to its palliative qualities [2]. 
Cardiac metastasis from squamous cancer cells of esophagus is a rare event which may appear during the evolution of this neoplasm. Heart metastases often present no clinical symptoms and are mostly diagnosed at autopsy [3, 4].

The pathophysiology of cardiac metastases is complex [5]. A tumor could spread to the heart via different paths such as direct cardiac extension, bloodstream, lymphatic system or intracavitary diffusion. Identification of the path of diffusion should be made on the basis of which cardiac structures are primarily affected: for example, myocardial involvement is the result of lymphatic spread, while endocardial metastases are the result of the heart chambers' being invaded through the bloodstream. The distinction regarding the metastatic pathway is based on a clinical evaluation of the structure of the heart involved or post-mortem examination [4]. Considering that most studies on the incidence of cardiac metastases are based on autopsies, as yet no in vivo evidence exists as to a possible correlation between hematogenous spread and cardiac metastases.

We report the case study of a 67-year-old man affected by a myocardial metastasis presenting as mimicking ST-segment elevation myocardial infarction. At cardiac progression, the patient underwent a peripheral venous blood sample collection to detect the presence of circulating tumor cells (CTCs) and to investigate their clinical significance.

\section{Case Report}

In September 2009, a 67-year-old Caucasian man had undergone definitive chemoradiation therapy for a locally advanced squamous cell cancer at the middle third of the esophagus. The patient had been suffering from a Child A cirrhosis and chronic bronchitis secondary to alcohol intake and tobacco consumption, respectively. At the end of the treatment, he had had a regular follow-up.

In May 2010, radiological examinations revealed the onset of bilateral lung metastases. The patient was admitted to our Department of Medical Oncology and was treated with 3 cycles of Al-Sarraf regimen with cisplatin and 5-fluorouracil (as first-line chemotherapy).

In August 2010, at the time of hospitalization, the patient complained of dyspnea and palpitations. At clinical evaluation, cardiac arrhythmia and hypotension (blood pressure $90 / 50 \mathrm{~mm} \mathrm{Hg}$ ) were detected. Chest radiography demonstrated an increase in cardiac silhouette. Electrocardiography (ECG) showed atrial fibrillation and ST-segment elevation from V3 to V6 derivations without Q waves (fig. 1). ECG did not change at subsequent controls. Values of troponin I and CK-MB (creatine-kinase musclebrain) were negative at baseline and subsequent controls.

Two-dimensional echocardiography revealed akinesia of the apex and septum of the heart, the presence of a mass at the apex of the left and right ventricular wall and a small amount of pericardial effusion. Left ventricular function appeared moderately impaired (ejection fraction was 40\%).

Cardiac magnetic resonance imaging (MRI) documented the presence of a mass of $40 \mathrm{~mm}$ in diameter in the apex and septum of the left ventriculum, the presence of pericardial effusion of $15 \mathrm{~mm}$ in thickness and disease progression to the lungs bilaterally (fig. 2).

The level of the tumor marker carcinoembryonic antigen was normal. The patient did not require surgical treatment. First-line chemotherapy was halted and the patient started supportive care. At discharge, ECOG performance score was 3. In January 2011, neoplastic effusion worsened and the patient died.

The patient's clinical history involved his having been enrolled in a prospective exploratory study to evaluate the presence of CTCs in peripheral blood. The local ethical committee had approved the study and the patient had provided written informed consent for inclusion into the study. CTC enumeration had been performed by a semi-automated system, namely CellSearch System (Veridex, LLC, Raritan, 
N.J., USA), which has been approved by the U.S. Food and Drug Administration for the isolation of CTCs in metastatic breast, colorectal and prostate cancer [6]. In these settings, CTCs above or below a cutoff level were proved to correlate to overall survival and disease progression. Experience derived from these studies led to the investigation of the possible applicability of the technique in different settings, such as esophageal cancer. In our case, a blood sample was drawn at the beginning of chemotherapy and at disease progression to the heart. The samples were collected in $10 \mathrm{ml}$ EDTA Vacutainer tubes, stored at room temperature and processed within $72 \mathrm{~h}$ of procurement. Samples were treated with a special mix of ferrofluids coated with epithelial cell-specific EpCAM antibodies to immunomagnetically enrich epithelial cells: a mixture of 2 phycoerythrin-conjugated antibodies that bound themselves to cytokeratins 8,18 , and 19; an antibody to CD45 conjugated to allophycocyanin; and nuclear dye (4,6-diamidino-2-phenylindole, DAPI) to label the cells fluorescently. Presence of a nucleus, expression of cytokeratins, cellular morphology and lack of CD45 expression were the characteristics required to define a CTC [7]. Results are expressed as the number of cells per $7.5 \mathrm{ml}$ of whole blood.

In our patient, no CTC was detected at the beginning of treatment. At cardiac progression, CTCs were 2 per $7.5 \mathrm{ml}$ of peripheral blood (fig. 3 ).

\section{Discussion}

The incidence of cardiac metastases reported in literature is variable ranging from 2.3 to $18.3 \%$, predominantly in the sixth and seventh decade of life [3]. Myocardial metastasis reflects systemic disease. Some tumors, like carcinomas of breast, lung, esophagus, malignant lymphoma and melanoma, diffuse preferentially to the heart [3]. Clinical presentation of cardiac involvement varies between the absence of symptoms and sudden death secondary to cardiac rupture, acute myocardial infarction or ventricular arrhythmias. ECG is often non-specific. Echocardiography is an ideal tool for diagnosis, yet MRI provides more specific anatomical information $[4,5]$.

Cardiac metastases have prognostic implications and therapies are mostly palliative $[2$, 4]. The therapeutic efficacy of a treatment must be balanced with its side effects and QOL; the approach should involve a multi-disciplinary team (medical oncologists, radiotherapists, surgeons, radiologists and cardiologists). The choice of treatment is conditioned by patient factors (performance status, age, comorbidities, organ dysfunction, patient preference), treatment factors (previous therapy, tolerability and response) and disease factors (type of tumor, tumor burden and tumor kinetics). In the case at hand, the patient did not undergo specific treatment in consideration of his previous therapy, tumor burden and performance status [2].

CTCs identified by the CellSearch System in metastatic breast cancer have been reported as having prognostic significance: the detection of $\geq 5$ cells per $7.5 \mathrm{ml}$ of blood before starting a systemic treatment is associated with a poorer prognosis [6]. This new system allows for the obtaining of highly accurate and reproducible results, as compared to other procedures.

Few studies have documented the presence of CTCs in metastatic esophageal cancer and their role in predicting survival. Allard et al. [7] reported the identification of CTCs in different types of cancer, gastrointestinal cancer included. In this report, the detection of $\geq 2$ CTCs per $7.5 \mathrm{ml}$ of blood by the CellSearch System was considered a positive result, after the evaluation of 964 patients with metastatic disease. More recently, Hiraiwa et al. [8] have reported the preliminary results of the utility of measuring CTCs in gastrointestinal cancer patients. They have confirmed the expression of EpCAM mRNA 
in gastrointestinal tumors, evaluating cancer cell lines: in particular, an esophageal cancer cell line was used to analyze the accuracy of the CellSearch assay. The authors investigated the role of CTCs in metastatic esophageal cancer and considered the unfavorable prognostic cutoff level at $\geq 2$ CTCs per $7.5 \mathrm{ml}$ of blood, based on the report by Allard et al. [7]. In this setting, they did not find a significant correlation between a positive CTC count and lung or liver metastases but the presence of $\geq 2$ CTCs per $7.5 \mathrm{ml}$ of blood had a significant correlation with serosal dissemination (pleural or peritoneal). The authors suggested that some CTCs might lack epithelial marker expression and may therefore not be detected by the system [8]. On the other hand, it could be hypothesized that the acquisition of these markers might play a prognostic role in cancer dissemination.

In our case report, we did not isolate circulating cells at baseline, when only lung metastases were detected. At the moment of cardiac involvement, 2 CTCs per $7.5 \mathrm{ml}$ of blood were found. In line with the description by Hiraiwa et al. [8], the myocardial and pericardial involvement seemed to correlate with the positive count of CTCs. The discovery of CTCs could be a possible explanation for the in vivo mechanism of cardiac spread: cancer cells may disseminate through the bloodstream to the heart. CTCs could play a part in the metastatic process and might be an independent marker of more aggressive cancer as well.

The identification of CTCs is still under investigation concerning both the method of detection and the significance of positive results. Prospective trials will be needed to confirm this hypothesis and to assess and characterize the clinical role of CTCs better.

\section{Disclosure Statement}

The authors have no conflicts of interest.

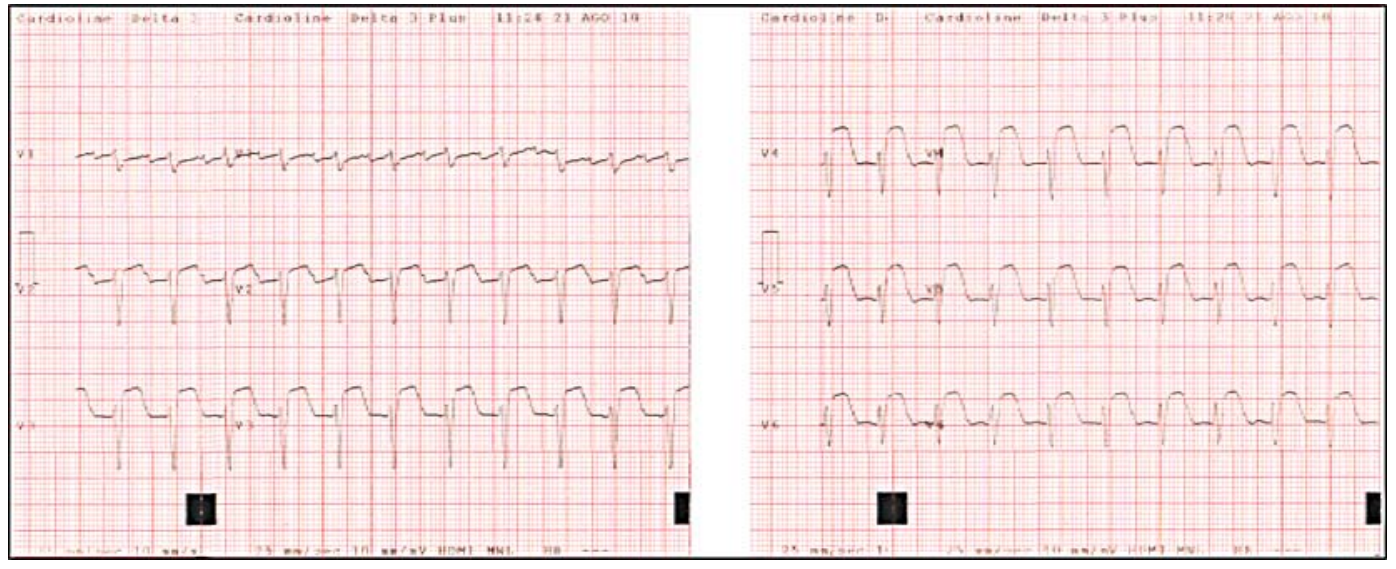

Fig. 1. Electrocardiography (ECG) showing atrial fibrillation and ST-segment elevation from V3 to V6 derivations without $\mathrm{Q}$ waves. 

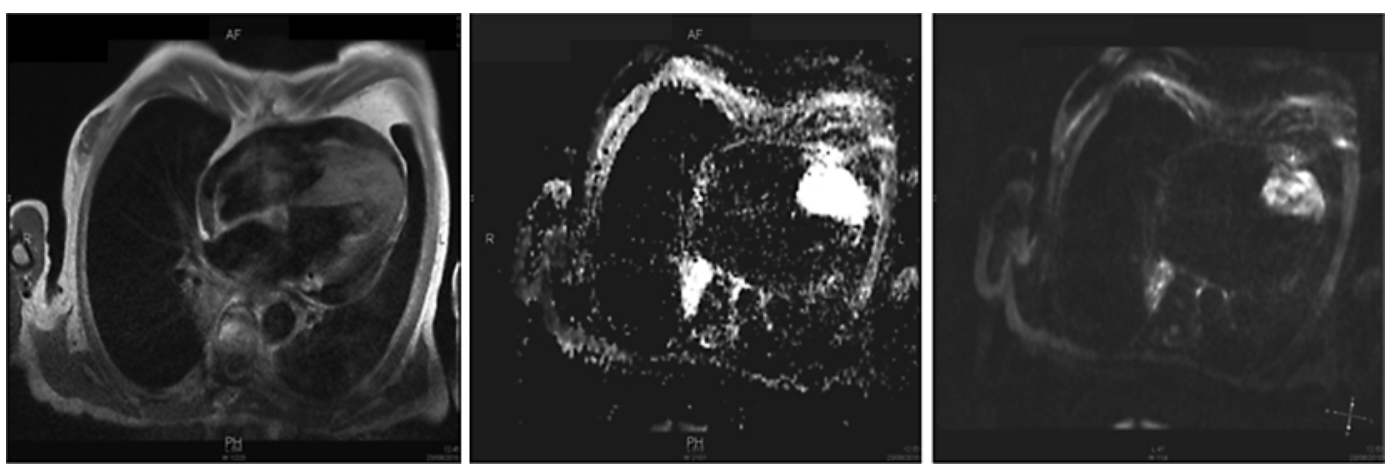

Fig. 2. MRI documented the presence of a mass of $40 \mathrm{~mm}$ in diameter in the apex and septum of the left ventriculum and the presence of pericardial effusion of $15 \mathrm{~mm}$ in thickness.

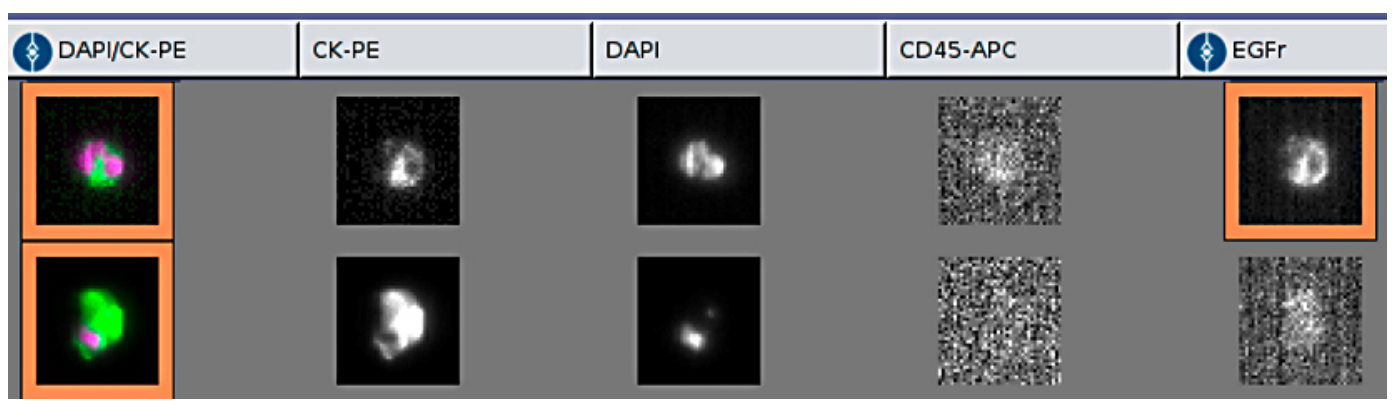

Fig. 3. Circulating tumor cells isolated by the CellSearch System in the peripheral blood. The 2 cells were cytokeratin (CK-PE)-, DAPI-, and EGFR-positive and CD45-negative.

\section{References}

-1 Bosetti C, Levi F, Ferlay J, Garavello W, Lucchini F, Bertuccio P, Negri E, La Vecchia C: Trend in oesophageal cancer incidence and mortality in Europe. Int J Cancer 2008;122:1118-1129.

2 Homs MY, Van Der Gaast A, Siersema PD, Steyerberg EW, Kuipers EJ: WITHDRAWN: chemotherapy for metastatic carcinoma of the esophagus and gastro-esophageal junction. Cochrane Database Syst Rev 2010;CD004063.

3 Reynen K, Kockeritz U, Strasser RH: Metastases to the heart. Ann Oncol 2004;15:375-381.

4 Maeda M, Goto T, Harigai M, Itoh T, Moriki T, Miyashita T: Myocardial metastasis from squamous cell carcinoma of the esophagus. Gen Thorac Cardiovasc Surg 2009;57:440-445.

5 Bussani R, De-Giorgio F, Abbate A, Silvestri F: Cardiac metastases. J Clin Pathol 2007;60:27-34.

-6 Cristofanilli M, Budd GT, Ellis MJ, Stopeck A, Matera J, Miller MC, Reuben JM, Doyle GV, Allard WJ, Terstappen LW, Hayes DF: Circulating tumor cells, disease progression and survival in metastatic breast cancer. N Eng J Med 2004;351:781-791.

7 Allard WJ, Matera J, Craig Miller M, Repollet M, Connelly MC, Rao C, Tibbe AGJ, Uhr JW, Terstappen LW: Tumor cells circulate in the peripheral blood of all major carcinomas but not in healthy subjects or patients with nonmalignant diseases. Clin Cancer Res 2004;10:6897-6904.

$\checkmark 8$ Hiraiwa K, Takeuchi H, Hasegawa H, Saikawa Y, Suda K, Ando T, Kumagai K, Irino T, Yoshikawa T, Matsuda S, Kitajima M, Kitagawa Y: Clinical significance of circulating tumor cells in blood from patients with gastrointestinal cancers. Ann Surg Oncol 2008;15:3092-3100. 